\title{
EPILOG
}

\section{Begrüßungs-Rundbrief von Joseph und Ema Lang an die Freunde und Genossen in der alten Heimat anläßlich ibrer Rückkebr aus den USA}

Hamburg, Ende August 1950

\section{Liebe Freunde und Genossen!}

Wir sind am 17. August in Bremen angekommen und von den dortigen Genossen so freundschaftlich und wohltuend in Empfang genommen worden, daß wir diese erste Begegnung mit den Freunden in der alten Heimat gern als verheißungsvollen Anfang ansehen wollen.

Als wir vor 16 Jahren aus Haft und Illegalität nach Prag flüchteten, ahnten wir nicht, welche Zeitspanne vor uns liegen und welche Ereignisse über uns alle hereinbrechen würden, bis wir wieder nach Deutschland zurückkehren können. Die schlimmsten Befürchtungen wurden übertroffen.

Und jetzt, s Jahre nach dem Zusammenbruch der Nazis, wird die gesamte Menschheit von der Furcht vor der Zukunft, von der Angst vor dem Kriege beherrscht. Wir kennen die Ursachen: Keines der Probleme, die unsere Generation seit 35 Jahren nicht mehr zur Ruhe kommen lassen, ist gelöst. Die sozialistischen Kräfte waren nicht in der Lage, entscheidend einzugreifen und im industriell entwickelten Teil Europas der Entwicklung ihren Stempel aufzudrücken.

Doch es ist nicht unsere Absicht, mit diesen ersten Zeilen große politische Ausführungen zu machen. Wir werden uns vermutlich in den nächsten Wochen und Monaten mit Euch über Entwicklungstendenzen und Perspektiven noch eingehend unterhalten. Wir möchten nur andeuten, daß nach unserer Meinung der allzu simple Traum vom friedlichen Hineinwachsen in den Sozialismus ebenso überwunden werden muß wie der Stalinismus mit seinem NKWD-Terror.

Heute wollen wir uns nur erst einmal bei Euch melden und Euch sagen, daß wir wohl wissen, welche Schwierigkeiten uns hier erwarten. Wir haben keine Illusionen. Wir glauben aber zutiefst, daß die Aufgaben, an deren Lösung wir seit Jahrzehnten mit Euch zusammenarbeiten, im Kern die gleichen geblieben sind.

Wir sind zurückgekommen, da wir nicht länger Zaungäste sein, sondern an Eurer Arbeit, Euren Versuchen und Euren Kämpfen teilnehmen wollen und um uns mit jenen Kräften zu verbinden, die gleich uns davon überzeugt sind, daß eine Regenerierung der Arbeiterbewegung ausgehen muß von den Erkenntnissen und Impulsen, von dem Ethos und dem Mut, die Karl Marx und Friedrich Engels ihr gaben. In diesem Sinne wollen wir uns wieder einreihen. Und wir vermuten, es wird gar nicht so schwer sein, uns mit Euch zu verständigen und eine gemeinsame Marschroute zu finden.

Wir kommen - trotz außenpolitischer Bedrohungen und unerfreulicher Anzeichen in innenpolitischer Hinsicht - mit Zuversicht und Freude, mit Toleranz und Aufgeschlossenheit, um wieder aktiven Anteil an Eurem Leben zu haben. Wir kommen ohne feste Pläne und wissen auch noch nicht, wo wir uns niederlassen werden. Um dies zu klären, um uns zu orientieren und uns mit dem Alltag vertraut zu machen, werden wir in einigen Wochen eine Rundreise durch Westdeutschland unternehmen, wobei wir voraussichtlich in alle wichtigen Zentren kommen werden.

Wir freuen uns, bei dieser Gelegenheit die persönliche Verbindung zu Euch herzustel- 
len. Von dem Ergebnis der Besprechungen mit Euch, von unseren Eindrücken und Erfahrungen wollen wir unsere Entscheidung, wo wir schließlich bleiben und was wir beginnen werden, abhängig machen.

Dies sei unser erster Gruß an Euch, liebe Freunde, wobei wir uns auch gern der Verpflichtung entledigen, Euch von den in Amerika verbliebenen Genossen, die mit uns gemeinsam an der Arbeit des Solidaritäts-Fonds beteiligt waren, zu grüßen und Euch

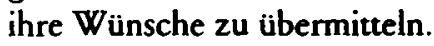

Ihr müßt entschuldigen, daß wir uns in der Form eines Zirkularbriefes an Euch wenden, aber der Freundeskreis ist so groß, daß es uns leider unmöglich ist, jedem von Euch separat zu schreiben. Wir schließen in der Hoffnung auf ein baldiges Wiedersehen und mit dem Wunsche auf ein gutes kameradschaftliches Zusammenwirken.

In herzlicher Verbundenheit und Freundschaft! Joseph und Erna Lang 
